\title{
Coherent Doppler Lidar Signal Covariance including Wind Shear and Wind Turbulence
}

\author{
R. G. Frehlich \\ Cooperative Institute for Research in the Environmental Sciences (CIRES) \\ University of Colorado, Boulder, CO 80309
}

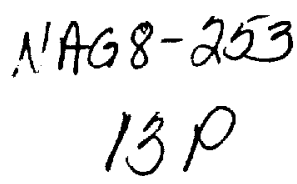

Abstract- The performance of coherent Doppler lidar is determined by the statistics of the coherent Doppler signal. The derivation and calculation of the covariance of the Doppler lidar signal is presented for random atmospheric wind fields with wind shear. The random component is described by a Kolmogorov turbulence spectrum. The signal parameters are clarified for a general coherent Doppler lidar system. There are two distinct physical regimes: one where the transmitted pulse determines the signal statistics and the other where the wind field dominates the signal statistics. The Doppler shift of the signal is identified in terms of the wind field and system parameters.

\section{Introduction}

Coherent Doppler lidar has become a promising new instrument for observations of the atmosphere. The first systems were based on the $\mathrm{CO}_{2} \operatorname{laser}^{1-4}$. More recently, solid state lasers have been successful integrated in Doppler lidar designs ${ }^{5-7}$. The targets for Dopper lidar are small atmospheric aerosol particles. Doppler lidar data can be the retum from a single pulse or a collection of many pulses along the same line of sight. The advantages of multiple pulse has been discussed by Rye and Hardesty ${ }^{8}$, and Frehlich ${ }^{9}$. Coherent Doppler lidar data is used to estimate the radial velocity of the targets using various mean-frequency estimators ${ }^{8-14}$.

The actual measurement quantity is related to the statistics of the random wind field over the sensing volume of the pulse. The effects of the pulse volume averaging for Doppler radar has been investigated by Doviak and Zmic ${ }^{15}$. The estimated Doppler frequency is defined in terms of the spectrum of the signal. Doppler radar data is generated by transmitting many pulses and sampling the retums at fixed sampling interval $T_{S}[s e c]$, which corresponds to sampling the same spatial volume defined by the pulse at equally spaced time intervals. For this case, the statistics of the data are determined by the time evol!?tion of the sensing volume of the pulse, and the spectrum is a well defined quantity. For coherent Doppler lidar, the data is generated from a single pulse as it travels through the atmosphere. The sensing volume of the measured Doppler frequency is determined by the distance the pulse travels in the finite observation time of each estimate $T=M T_{S}$, where $M$ is the total number of data points per estimate. The spectrum is not well defined and the Doppler frequency is related to the statistics of the wind field over the sensing volume of the single pulse. The estimated parameters are then related to the wind fields and lidar system parameters in a different fashion than for the Doppler radar. This connection will be derived to clarify the meaning of coherent Doppler lidar measurements and pave the way for more reliable estimators of the signal parameters.

The statistical description of coherent Doppler lidar data from aerosol targets has been shown to be well represented as a zero mean Gaussian random process. It is a common practice to follow the tradition of Doppler radar and convert the real signal to the complex analytical signal using a quadrature receiver ${ }^{15-17}$. This generates complex data with desirable statistical properties that simplify the calculation of the Cramer-Rao Bound ${ }^{14,13,18}$ (the ideal performance of estimators of the signal parameters) and the development of efficient estimators of the signal parameters. The statistical description of the data is required for these analysis. Since the data is a Gaussian random process, the signal covariance provides a complete statistical description of the data. The signal covariance is derived for a general coherent Doppler lidar including random wind fields and wind shear. The derivation follows the analyses of Frehlich and Kavaya ${ }^{19}$ for the performance of coherent laser radar for general atmospheric refractive turbulence.

\section{Coherent Doppler Lidar Signal}

A coherent Doppler lidar can be represented by the transmitter aperture function $W_{T}(\bar{U})$, the transmitter laser field incident on the transmitter aperture $E_{L}(\vec{u}, 0, t)$, the receiver aperture function $W_{R}(\vec{v})$, and the Local Oscillator $(\mathrm{LO})$ field incident on the receiver aperture $E_{L O}(\vec{\nabla}, 0)$. The transmitter laser field 
with frequency $f_{L}[\mathrm{~Hz}]$ is given by

$$
E_{L}(\vec{u}, 0, t)=A_{L}(t) e_{L}(\vec{u}, 0) \exp \left(2 \pi i f_{L} t\right)
$$

where $t[s]$ denotes time, $e_{L}(\overrightarrow{\mathrm{u}}, 0)$ is the normalized spatial field which is assumed independent of time $t$ (the spatial mode of the laser pulse is independent of time) and

$$
A_{L}(t)=P_{L}^{1 / 2}(t) \exp \left[2 \pi i \int_{0}^{1} f_{C}\left(t^{\prime}\right) d t^{\prime}\right]
$$

is the temporal behavior of the complex amplitude of the pulse where $P_{L}(t)[W]$ is the pulse power and $f_{C}\left(t^{\prime}\right)[\mathrm{Hz}]$ is the frequency chirp of the transmitted pulse. The LO field in the receiver plane with frequency $f_{L O}[\mathrm{~Hz}]$ is given by

$$
E_{L O}(\vec{\nabla}, 0)=P_{L O}^{1 / 2} e_{L O}(\vec{\nabla}, 0) \exp \left(2 \pi i f_{L O} t\right)
$$

where $P_{L O}[W]$ is the LO power and

$$
\int_{-\infty}^{\infty}\left|e_{L L O}(\overrightarrow{\mathrm{u}}, 0)\right| d \overrightarrow{\mathrm{u}}=1
$$

The coherent Doppler lidar typically operates under the ideal condition of quantum limited detection, i.e., the signal noise is dominated by the shot noise of the LO field. We will assume that the detector quantum efficiency $\eta_{Q}$ is uniform over the surface of the detector and that all the LO power is collected by the detector.

For Doppler lidar measurements of the atmosphere, the backscattered field is a zero-mean Gaussian random process because it is the superposition of all the backscattered fields from many random aerosols is the superposition of the backscattered field from many aerosol particles. The doppler lidar signal is also a zero-mean Gaussian random process. It is convenient to normalize the signal by the standard deviation of the noise signal and employ the complex representation (Appendix A)

$$
Z(t)=S(t)+N(t)=\operatorname{Re}[z(t)]
$$

where $S(t)$ is the normalized Doppler lidar IF current signal from the backscattered field and $N(t)$ is the nurmalized additive detector noise. The statistical description of a Gaussian random process is given by the covariance function

$$
\begin{gathered}
B_{Z}\left(t_{1}, t_{2}\right)=<Z\left(t_{1}\right) Z\left(t_{2}\right)>=\operatorname{Re}\left[R_{z}\left(t_{1}, t_{2}\right)\right] \\
R_{z}\left(t_{1}, t_{2}\right)=\frac{1}{2}\left\langle z\left(t_{1}\right) z^{*}\left(t_{2}\right)>\right. \\
<z\left(t_{1}\right) z\left(t_{2}\right)>=0
\end{gathered}
$$

where the ensemble average $<>$ is performed over the appropriate random process. With this normalization

$$
B_{z}\left(t_{1}, t_{2}\right)=\delta\left(t_{1}-t_{2}\right)+B_{S}\left(t_{1}, t_{2}\right), \quad R_{2}\left(t_{1}, t_{2}\right)=\delta\left(t_{1}-t_{2}\right)+R_{s}\left(t_{1}, t_{2}\right)
$$

where $B_{S}\left(t_{1}, t_{2}\right)$ and $R_{s}\left(t_{1}, t_{2}\right)$ are the covariance of the normalized Doppler lidar signals,

$$
B_{S}(t, t)=R_{S}(t, t)=\operatorname{SNR}(t)
$$

and $S N R$ is the coherent lidar signal-to-noise ratio with respect to the ensemble average. The Doppler lidar signal is typically uncorrelated from shot to shot because the aerosol targets move more than a wavelength between shots, which produces random phases for the backscattered signal. The ensemble average over the aerosol particles is the most common ensemble average and produces (Appendix A)

$R_{S}\left(t_{1}, t_{2}\right)=\frac{\eta_{Q}}{h \vee B} \int_{0}^{\infty} \int_{-\infty}^{\infty} A_{L}\left(t_{1}-2 \frac{z}{c}\right) A_{L}^{*}\left(t_{2}-2 \frac{z}{c}\right) K^{2}(\overrightarrow{\mathrm{p}}, z) \beta(\overrightarrow{\mathrm{p}}, z)|Q(\overrightarrow{\mathrm{p}}, z)|^{2} \exp \left\{2 \pi i \tau\left[\Delta F-\frac{2}{\lambda} v_{r}(\overrightarrow{\mathrm{p}}, z)\right]\right\} d \overrightarrow{\mathrm{p}} d z$

where $v_{r}(\overrightarrow{\mathbf{p}}, z)$ is the radial component of the velocity of the aerosol particles at coordinate $(\overrightarrow{\mathbf{p}}, z)$, $\Delta F=f_{L}-f_{L O}$ is the frequency difference between the transmitted field and the LO field, and 


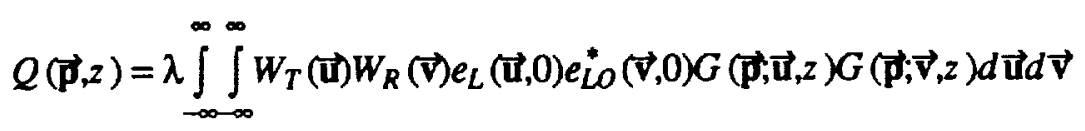

where $G(\vec{p}, \mathbf{u}, z)$ is the Green's function for propagating the field from the transmitter plane to $(\boldsymbol{p}, z)$. For Doppler lidar, the complex signal $z(t)$ is also an analytic signal because Eq. (8) is satisfied (see Appendix A). This provides simplifications for data analysis and estimation algorithms. The function $R\left(t_{1}, t_{2}\right)$ is a hermitian function, i.e.,

$$
R\left(t_{1}, t_{2}\right)=R^{*}\left(t_{2}, t_{1}\right)
$$

With a high pulse rate Doppler lidar, many shots can be transmitted during the temporal scale of the random Green's function $G(\vec{p} ; \vec{u}, z)$, which is usually determined by the atmospheric refractive turbulence. This permits investigations into the statistics of refractive turbulence. The ensemble average over refractive turbulence produce

$$
\begin{gathered}
R_{S}\left(t_{1}, t_{2}\right)=\frac{\eta_{Q}}{h \vee B} \int_{0-\infty}^{\infty} \int_{-\infty}^{\infty} A_{L}\left(t_{1}-2 z / c\right) A_{L}^{*}\left(t_{2}-2 z / c\right) O(\overrightarrow{\mathbf{p}}, z) \exp \left\{2 \pi i \tau\left[\Delta F-2 v_{r}(\overrightarrow{\mathrm{p}}, z) / \lambda\right]\right\} d \overrightarrow{\mathbf{p}} d z, \\
O(\overrightarrow{\mathbf{p}}, z)=K^{2}(\overrightarrow{\mathbf{p}}, z) \beta(\overrightarrow{\mathbf{p}}, z) c(\overrightarrow{\mathbf{p}}, z),
\end{gathered}
$$

where

$$
c(\overrightarrow{\mathbf{p}}, z)=|Q(\overrightarrow{\mathbf{p}}, \mathbf{z})|^{2},
$$

is the coherent responsivity density ${ }^{19}$ of the coherent Doppler lidar. For many applications, the effects of refractive turbulence are negligible and

$$
G(\vec{p} ; \overrightarrow{\mathrm{u}}, z)=G^{f}(\overrightarrow{\mathrm{p}}, \overrightarrow{\mathrm{u}}, \mathbf{z})=\frac{i k}{2 \pi z} \exp \left[-\frac{i k}{2 z}(\overrightarrow{\mathrm{p}}-\overrightarrow{\mathrm{u}})^{2}\right]
$$

where $G^{f}(\overrightarrow{\mathbf{p}} ; \overrightarrow{\mathrm{u}}, z)$ is the free space Green's function and $k=2 \pi / \lambda\left[m^{-1}\right]$ is the wavenumber of the laser field. The fluctuations of the wind field $\vec{\nabla}(\vec{p}, z)$, atmospheric extinction $K(\overrightarrow{\mathbf{p}}, z)$, and backscatter coefficient $\beta(\vec{p}, z)$, are the remaining random quantities.

A typical Doppler lidar has a narrow beam compared to the dimensions of the range gate. Then, the dependence on the transverse coordinate $\mathbf{p}$ is small and

$$
R_{S}\left(t_{1}, t_{2}\right)=\frac{\eta_{Q}}{h \vee B} \int_{0}^{\infty} H(z) A_{L}\left(t_{1}-2 z / c\right) A_{L}^{*}\left(t_{2}-2 z / c\right) \exp \left\{2 \pi i \tau\left[\Delta F-2 v_{r}(0, z) / \lambda\right]\right\} d z
$$

where

$$
H(z)=K^{2}(0, z) \beta(0, z) C(z)
$$

is the system gain and

$$
C(\mathrm{z})=\int_{-\infty}^{\infty} c(\overrightarrow{\mathbf{p}}, \mathrm{z}) d \overrightarrow{\mathbf{p}}
$$

is the coherent responsivity ${ }^{19}$ of the coherent Doppler lidar.

The center of a range gate for Doppler lidar measurements is defined by

$$
z_{0}=c t_{d} 2
$$

and the total observation time corresponding to this range gate is $\left(t_{\sigma}-T / 2, t_{0}+T / 2\right)$. If $\beta(\vec{p}, z), K(\vec{p}, z)$, $v_{r}(\mathbf{p}, z)$, and $c(\mathbf{p}, z)$ are constant over the sensing volume of this range gate and if there is no frequency chirp $f_{C}(t)$, we have for all $\left(t_{1}, t_{2}\right)$ in the observation interval

$$
R_{S}\left(t_{1}, t_{2}\right)=R_{S}(\tau)=\frac{\eta_{Q} c}{2 h \vee B} H\left(z_{0}\right) \exp (2 \pi i \tau F) \int_{-\infty}^{\infty}\left[P_{L}(t) P_{L}(t-\tau)\right]^{1 / 2} d t
$$

where 


$$
F=\Delta F-2 v_{r}\left(0, z_{0}\right) / \lambda
$$

is the mean frequency of the Doppler lidar signal, $\tau=t_{1}-t_{2}$ and the limits of integration have been extended to infinity. The signals $x(t)$ and $Z(t)$ are stationary over the observation interval $\left(t_{0}-T / 2, t_{0}+T / 2\right)$. For a Gaussian pulse with

$$
\begin{gathered}
P_{L}(t)=P_{0} \exp \left(-t^{2} / \sigma^{2}\right) \\
R_{S}(\tau)=S N R\left(z_{0}\right) \exp \left(2 \pi i F-\tau^{2} / \tau_{P}{ }^{2}\right)
\end{gathered}
$$

where

$$
\operatorname{SNR}\left(\mathrm{z}_{0}\right)=\frac{\eta_{Q} c U_{L}}{2 h v B} H\left(z_{0}\right)
$$

is the SNR for a pulse centered at range $z_{0}$,

$$
U_{L}=\int_{-\infty}^{\infty} P_{L}(t) d t
$$

is the pulse energy and $\tau_{P}=2 \sigma$ is the decorrelation time of the lidar signal for uniform wind fields.

The signal spectrum is defined as

$$
\Phi_{z}(f)=\int_{-\infty}^{\infty} R_{z}(\tau) \exp (-2 \pi i \tau f) d \tau
$$

and

$$
\Phi_{z}(f)=\frac{\pi \eta_{\ell} c}{h \vee B} K^{2}\left(z_{0}\right) \beta\left(z_{0}\right) C\left(z_{0}\right) M(f-F)
$$

where

$$
M(f)=\left|\int_{-\infty}^{\infty} P_{L}(t)^{1 / 2} \exp (-2 \pi i f t) d t\right|^{2}
$$

is the spectrum of the transmitted pulse. The signal spectrum is also the same as the signal spectrum from a rigid or diffuse hard target.

\section{Effects of Wind Shear}

Doppler lidar data permits estimation of the radial component of the wind field $v_{r}(\overrightarrow{\mathbf{p}}, z)$ with a single shot. For short pulse duration, the random fluctuations of $v_{r}(\vec{p}, z)$ can be approximated by the first two terms of it's Taylor series expansion in z, i.e.,

$$
v_{r}(\boldsymbol{p}, z)=v_{r}\left(\mathbf{p}, z_{0}\right)+g\left(\mathbf{p}, z_{0}\right)\left(z-z_{0}\right)
$$

where $g\left(\vec{p}, z_{0}\right)\left[s^{-1}\right]$ is the wind shear of the radial component of the velocity at $\left(\vec{p}, z_{0}\right)$, i.e.,

$$
g(\overrightarrow{\mathbf{p}}, z)=\frac{\partial v_{r}(\mathbf{p}, z)}{\partial z}
$$

The range dependent system gain $O(\vec{p}, z)$ is approximated by the first two terms of it's Taylor series expansion in $z$, i.e.,

$$
O(\overrightarrow{\mathbf{p}}, z)=O\left(\overrightarrow{\mathbf{p}}, z_{0}\right)\left[1+b\left(\overrightarrow{\mathbf{p}}, z_{0}\right)\left(z-z_{0}\right)\right]
$$

where

$$
b(\overrightarrow{\mathbf{p}}, z)=\frac{1}{O(\overrightarrow{\mathbf{p}}, z)} \frac{\partial O(\overrightarrow{\mathbf{p}}, z)}{\partial z}
$$

When these approximations are valid and for a Gaussian pulse [see Eq. (24)] 


$$
\begin{aligned}
& R_{S}\left(t_{1}, t_{2}\right)=\frac{\eta_{Q} c U_{L}}{2 h v B} \int_{-\infty}^{\infty} O\left(\overrightarrow{\mathbf{p}}, z_{0}\right)\left[1+2 b\left(\overrightarrow{\mathbf{p}}, z_{0}\right) \Delta r\left(\mu / \sigma-t \alpha \sigma-i \frac{\tau}{2 \tau_{W S}}\right)\right] \\
& \exp \left\{2 \pi i \tau\left[\Delta F-2 v_{r}(\overrightarrow{\mathbf{p}}, z) / \lambda+2\left(t_{\sigma}-\mu\right) /\left(\tau_{W S} \sigma\right)\right]-\tau^{2} / \tau_{P}{ }^{2}-\tau^{2} / \tau_{W S}{ }^{2}\right\} d \overrightarrow{\mathbf{p}}
\end{aligned}
$$

where $\mu=\left(t_{1}+t_{2}\right) / 2$,

$$
\tau_{W S}=2 \frac{\lambda}{g\left(\overrightarrow{\mathbf{p}}, z_{0}\right) \pi \Delta r}
$$

is the decorrelation time due to wind shear and

$$
\Delta r=c \sigma / 2
$$

is the length of the pulse in range or the range resolution. For a Doppler lidar with a narrow beam compared to the dimensions of the range gate

$$
\begin{gathered}
R_{S}\left(t_{1}, t_{2}\right)=S N R\left(z_{0}\right)\left[1+2 \gamma\left(\mu / \sigma-t_{0} / \sigma-i \frac{\tau}{2 \tau_{W S}}\right)\right] \\
\exp \left\{i \tau\left[2 \pi F-2\left(\mu-t_{0}\right) /\left(\tau_{W S} \sigma\right)\right]-\tau^{2} / \tau_{P}{ }^{2}-\tau^{2} / \tau_{W S}{ }^{2}\right\}
\end{gathered}
$$

where

$$
\gamma=b\left(0, z_{0}\right) \Delta r=\left[O\left(0, z_{0}+\Delta r\right)-O\left(0, z_{0}\right)\right] / O\left(0, z_{0}\right)
$$

is the fractional change of the gain $O(0, z)$ over the range resolution $\Delta r$ at $z_{0}$, if the linear approximation of Eqs. (31) and (33) are valid.

The rate of decay of the covariance is dominated by the wind shear when

$$
2 \pi c \sigma^{2} g\left(0, z_{0}\right) / \lambda \gg 1
$$

This occurs when the extra Doppler shift due to the wind shear at a separation of the range resolution $\Delta r$ is larger than the spectral width of the signal due to the pulse profile only [see Eq. (29)]

The effects of wind shear with constant SNR $[H(z)]$ over the observation interval are shown in Fig. 1. The wind shear produces a rapid decorrelation of the lidar signal, which appears as a damped oscillating signal as the observation points $t_{1}$ and $t_{2}$ deviate from the center of the observation interval $t_{0}$. The effects of a gradient in the SNR $[H(z)]$ is shown in Fig. 2. The gradient produces an amplitude change in addition to the effects of wind shear.

\section{Effects of Wind Turbulence}

Atmospheric wind fields are characterized by random fluctuations in space and time. For many cases, the statistics of the wind fields are well characterized by universal functions of basic parameters. For Doppler radar the effects of the random fluctuations of the wind field over the sensing volume has been discussed by Doviak and $\mathrm{Zmic}^{15}$. For Doppler lidar, the situation is complicated because estimates of the velocity are computed for a single shot. Ensemble averages over many shots was considered by Churnside and Yura ${ }^{21}$. The effects of spatial variations of backscatter coefficient over the sensing volume of the pulse were investigated by Rye using simulations 22 .

The covariance of the Doppler lidar signal for a single shot is given by Eq. (14), which can be written as

$$
R_{S}\left(t_{1}, t_{2}\right)=\frac{\eta_{Q}}{h v B} \int_{0}^{\infty} \int_{-\infty}^{\infty} A_{L}\left(t_{1}-2 z / c\right) A_{L}^{*}\left(t_{2}-2 z / c\right) O\left((\overrightarrow{\mathbf{p}}, z) \exp \left\{2 \pi i \tau\left[F-2 \Delta v_{r}(\overrightarrow{\mathbf{p}}, z) / \lambda\right]\right\} d z\right.
$$

where

$$
\Delta v_{r}\left(\overrightarrow{\mathbf{p}}, z_{,}, z_{0}\right)=v_{r}(\overrightarrow{\mathbf{p}}, z)-v_{r}\left(0, z_{0}\right)
$$


is the difference of the radial component of the velocity compared to $v_{r}\left(0, z_{0}\right)$. The mean frequency $f$ is a random variable because the wind field is random. The estimation error for estimates of the mean frequency $F$ using data from a single shot depends on the statistical properties of $\Delta v_{r}\left(\overline{\mathbf{p}}, z_{,}, z_{0}\right)$ and the function $H(\vec{p}, z)$. For well behaved atmospheric conditions, the velocity difference $\Delta v_{r}\left(\vec{p}, z, z_{0}\right)$ is approximately a zero mean Gaussian random variable. Performing the ensemble average over this random variable produces

$$
R_{S}\left(t_{1}, t_{2}\right)=\frac{\eta_{Q}}{h v B} \int_{0}^{\infty} \int_{-\infty}^{\infty} A_{L}\left(t_{1}-2 z / c\right) A_{L}^{*}\left(t_{2}-2 z / c\right) H(\overline{\mathbf{p}}, z) \exp \left[2 \pi i \tau F-2 \tau^{2} D_{r}\left(\overline{\mathbf{p}}, z, z_{0}\right) / \lambda^{2}\right] d z
$$

where

$$
D_{r}\left(\mathbf{p}, z, z_{0}\right)=\left\langle\Delta v_{r}\left(\mathbf{p}, z, z_{0}\right)^{2}\right\rangle
$$

is the structure function of the fluctuations of the radial component of the velocity field. When the statistics of the velocity fields over the sensing volume of the pulse are isotropic ${ }^{20}$

$$
D_{r}\left(\overrightarrow{\mathbf{p}}, z_{,}, z_{0}\right)=C_{v} \varepsilon^{2 / 3}\left(z_{0}\right) r^{2 / 3}\left[\left(z-z_{0}\right)^{2}+4 p^{2} / 3\right] / r^{2}
$$

where $C_{v}$ is the Kolmogorov constant, $\varepsilon\left[m^{2} s^{-2}\right]$ is the energy dissipation rate, and $r^{2}=\left(z-z_{0}\right)^{2}+p^{2}$. For a Guassian transmitted pulse

$$
\begin{gathered}
R_{S}\left(t_{1}, t_{2}\right)=\frac{\eta_{Q} U_{L} c \Delta r^{2}}{2 \pi^{1 / 2} h v B} \exp \left(2 \pi i \tau F-\tau^{2} / \tau_{P}{ }^{2}\right) \\
\int_{-\infty}^{\infty} \int_{-\infty}^{\infty} H\left(\Delta r \overrightarrow{\mathrm{q}}, \Delta r \zeta+z_{0}\right) \exp \left\{-\left[\left(\mu-t_{0}\right) / \sigma-\zeta\right]^{2}-8 \tau^{2} \Lambda \kappa^{2 / 3}\left(\zeta^{2}+4 q^{2} / 3\right) /\left(\kappa \tau_{P}\right)^{2}\right\} d \overrightarrow{\mathrm{q}} d \zeta
\end{gathered}
$$

where $\kappa^{2}=\zeta^{2}+q^{2}$ and

$$
\Lambda=\sigma^{2} C_{\nu}(\varepsilon \Delta r)^{2 / 3} / \lambda^{2}=\sigma^{2} D_{r}\left(0, z_{0}+\Delta r, z_{0}\right) / \lambda^{2}
$$

represents the contribution from wind turbulence.

For a coherent Doppler lidar with a Gaussian transmitted spatial profile ${ }^{19}$

$$
H\left(\Delta r \overrightarrow{\mathrm{q}}, z_{0}\right)=\frac{C\left(z_{0}\right)}{\pi \sigma_{B}^{2}\left(z_{0}\right)} \exp \left[-\Delta r^{2} q^{2} / \sigma_{B}^{2}\left(z_{0}\right)\right]
$$

where $C\left(z_{0}\right)$ is the coherent responsivity at range $z_{0}$ and $\sigma_{B}\left(z_{0}\right)$ is the $1 / e$ intensity radius of the transmitted beam at range $z_{0}$. For many cases of interest, $C\left(z_{0}\right)$ and $\sigma_{B}$ are constant with range over the sensing volume of the pulse and

$$
\begin{gathered}
R_{S}\left(t_{1}, t_{2}\right)=\frac{2 S N R\left(t_{0}\right) \Delta r^{2}}{\pi^{1 / 2} \sigma_{B}^{2}} \exp \left(2 \pi i \tau F-\tau^{2} / \tau_{P}{ }^{2}\right) \\
\int_{-\infty}^{\infty} \int_{-\infty}^{\infty} \exp \left\{-\left[\left(\mu-t_{0}\right) / \sigma-\zeta\right]^{2}-8 \tau^{2} \Lambda \kappa^{2 / 3}\left(\zeta^{2}+4 q^{2} / 3\right) /\left(\kappa \tau_{P}\right)^{2}\right\} d \vec{q} d \zeta
\end{gathered}
$$

For a narrow beam, the integration over $\vec{q}$ can be performed and

$$
R_{S}\left(t_{1}, t_{2}\right)=\frac{S N R\left(z_{0}\right)}{\pi^{1 / 2}} \exp \left(2 \pi i \tau F-\tau^{2} / \tau_{P}{ }^{2}\right) \int_{-\infty}^{\infty} \exp \left\{-\left[\left(\mu-t_{0}\right) / \sigma-\zeta\right]^{2}-8 \tau^{2} \Lambda \zeta^{2 / 3} / \tau_{P}{ }^{2}\right\} d \zeta
$$

The effects of wind turbulence and the finite transverse dimensions of the beam is shown in Fig. 3 . The decrease in the correlation scale due to turbulence is more pronounced for a wide beam $\left(\sigma_{B}>\Delta r\right)$ because of the increase in the Doppler shifts from the random fluctuations of the wind in the transverse dimensions.

The effects of wind turbulence for the common case of a narrow transmitted beam compared to the range resolution $\left(\sigma_{B}<\Delta r\right)$ is shown if Fig. 4 . 


\section{Summary}

The signal covariance for coherent Doppler lidar data is derived including a linear wind shear and linear system gain over the sensing volume of the pulse. Results are also presented for the case of isotropic and homogeneous wind turbulence. For both these case, the signal statistics are not stationary over the observation interval. The use of estimators for the system parameters that assume stationary (spectral domain estimators) may not perform efficiently in these regimes. Improved performance is possible using the improved description of the data contained in the signal covariance.

\section{Acknowledgement.}

The author acknowledges useful discussions with M. J. Kavaya and B. J. Rye. This work was supported by the National Science Foundation and the National Aeronautics and Space Administration.

\section{APPENDIX A: Coherent Doppler Lidar Signals}

The total coherent or heterodyne Doppler lidar signal $i_{T}(t)[A]$ is the sum of the Doppler IF signal current $i_{S}(t)[A]$ and the detector noise current $i_{N}(t)[A]$ which is conveniently written in complex notation as $i_{T}(t)=\operatorname{Re}[Y(t)], i_{N}(t)=\operatorname{Re}[U(t)]$, where

$$
Y(t)=U(t)+\frac{2 G_{D} e}{h \mathrm{v}} \int_{b} \eta_{Q}(\overrightarrow{\mathrm{w}}) E_{S}(\overrightarrow{\mathrm{w}}, L, t) E_{L O}^{*}(\overrightarrow{\mathrm{w}}, L) \exp \left(i \Delta \omega t+i \theta_{S}\right) d \overrightarrow{\mathrm{w}}
$$

$G_{D}$ is the dimensionless amplifier gain, $e=1.602 \times 10^{-19}[C / e l e c t r o n]$ is the electronic charge, $h=6.626 \times 10^{-34}[\mathrm{Js}]$ is Planck's constant, $v[\mathrm{~Hz}]$ is the optical frequency, $\eta_{Q}(\vec{w})$ [electrons/photon] is the detector quantum efficiency function on the detector surface, $E_{S}(\vec{w}, L, t)$ is the backscattered field on the detector surface, $E_{L O}(\vec{w}, L)$ is the LO field on the detector surface, $\theta_{S}[\mathrm{rad}]$ is the random phase of the backscattered field compared to the LO field, $\Delta \omega[\mathrm{rad} / \mathrm{sec}]$, is the angular frequency difference between the transmitted and LO field, $\overrightarrow{\mathbf{w}}[m]$ is the transverse coordinate on the detector surface and $d \overrightarrow{\mathrm{w}}$ denotes two-dimensional integration over the detector surface. For ideal optical systems and for a infinite uniform detector, the fields on the detector can be represented in terms of the fields in the plane of the receiver optics, i.e.,

$$
Y(t)=U(t)+\int_{-\infty}^{\infty} E_{S}(\vec{\nabla}, 0, t) E_{L O}^{*}(\vec{\nabla}, 0) W_{R}(\vec{\nabla}) \exp \left(i \Delta \omega t+i \Theta_{S}\right) d \vec{\nabla}
$$

where $E_{S}(\vec{\nabla}, 0, t)$ and $E_{L O}(\vec{\nabla}, 0)$ are the backscattered field and $L O$ field in the receiver plane.

If the signal noise is dominated by the shot noise generated by the local oscillator field (quantum limited detection), the average noise power is

$$
\left\langle i_{N}^{2}(t)\right\rangle=\frac{1}{2}\left\langle U(t) U^{*}(t)\right\rangle=2 G_{D}^{2} e^{2} B \eta_{Q} P_{L O} /(h v)
$$

where $B[\mathrm{~Hz}]$ is the noise bandwidth. It is convenient to normalize the signals by the rms of the noise current, i.e.,

$$
Z(t)=Y(t) /<i_{N}^{2}(t)>^{1 / 2}=S(t)+N(t) .
$$

The backscattered field from a point scatterer at coordinate $(\overrightarrow{\mathbf{p}}, z)$ with scattering cross section $\sigma_{S}$ is

$$
E_{S}(\overrightarrow{\mathbf{v}}, 0, t)=\lambda \sigma_{S}^{1 / 2} K(\overrightarrow{\mathbf{p}}, z) \int_{-\infty}^{\infty} E_{L}(\overrightarrow{\mathbf{u}}, 0, t-2 z / c) W_{T}(\overrightarrow{\mathbf{u}}) G(\overrightarrow{\mathbf{p}} ; \overrightarrow{\mathbf{u}}, z) G(\overrightarrow{\mathbf{p}} ; \overrightarrow{\mathbf{v}}, z) \exp \left[i \Theta(\overrightarrow{\mathbf{p}}, z)-4 \pi i t v_{r}(\overrightarrow{\mathbf{p}}, z) / \lambda\right] d \overrightarrow{\mathbf{u}}
$$

where $\Theta(\vec{p}, z)$ is the random phase of the backscattered field. The total backscattered field is the sum of all the backscattered fields from the aerosol particles illuminated by the transmitted field. Substituting the total backscattered field into Eqs. (A2), (A4), (7), (1), performing the ensemble average over the random phases $\theta(\bar{p}, z)$ using the identity $<\exp \left[i\left(\theta\left(p_{1}, z_{1}\right)+\theta\left(p_{2}, z_{2}\right)\right]>=0\right.$, and converting the summation over aerosol particles to integration ${ }^{19}$ produces Eq. (11). The same procedure produces Eq. (8). 


\section{REFERENCES}

1. J. W. Bilbro, C. DiMarzio, D. Fitzjarrald, S. Johnson, and W. Jones, "Airborne Doppler lidar measurements," Appl. Opt. 25, 2952-2960 (1986).

2. J. C. Petheram, G. Frohbeiter, and A. Rosenberg, "Carbon dioxide doppler lidar wind sensor on a space station polar platform," Appl. Opt. 28, 834-839 (1989).

3. M. J. Post and R. E. Cupp, "Optimizing a pulsed doppler lidar," Appl. Opt. 29, 4145-4158 (1990).

4. G. N. Pearson and B. J. Rye, "Frequency fidelity of a compact $\mathrm{CO}_{2}$ Doppler lidar transmitter," Appl. Opt. 31, 6475-6484 (1992).

5. M. J. Kavaya, S. W. Henderson, J. R. Magee, C. P. Hale, and R. M. Huffaker, "Remote wind profiling with a solid-state nd:yag coherent lidar system," Opt. Let. 14, 776-778 (1992).

6. S. W. Henderson, C. P. Hale, J. R. Magee, M. J. Kavaya, and A. V. Huffaker, "Eye-safe coherent laser radar system at $2.1 \mu \mathrm{m}$ using Tm,Ho:YAG lasers," Opt. Lett. 16, 773-775 (1991).

7. S. W. Henderson, P. J. M. Suni, C. P. Hale, S. M. Hannon, J. R. Magee, D. L. Bruns, and E. H. Yuen, "Coherent laser radar at $2 \mu \mathrm{m}$ using solid-state lasers," IEEE Trans. Geo. Remote Sensing 31, 4-15 (1993).

8. B. J. Rye, and R. M. Hardesty, "Discrete spectral peak estimation in incoherent backscatter heterodyne lidar. I. Spectral accumulation and the Cramer-Rao lower bound," IIEEE Trans. Geo. Sci. Remote Sensing 31, 16-27 (1993).

9. R. M. Hardesty, "Performance of a discrete spectral peak frequency estimator for Doppler wind velocity measurements," IEEE Trans. Geosci. Remote Sensing GE-24, 777-783 (1986).

10. P. R. Mahapatra and D. S. Zrnic, "Practical algorithms for mean velocity estimation in pulse Doppler weather radars using a small number of samples," IEEE Trans. Geosci. Electronics GE-21, 491-501 (1983).

11. P. T. May and R. G. Strauch, "An examination of Wind Profiler signal processing algorithms," Atmos. Oceanic Tech. 6, 731-735 (1989).

12. P. T. May, T. Sato, M. Yamamoto, S. Kato, T. Tsuda, and S. Fukao, "Errors in the determination of wind speed by Doppler radar," Atmos. Oceanic Tech. 6, 235-242 (1989).

13. B. J. Rye and R. M. Hardesty, "Discrete spectral peak estimation in incoherent backscatter heterodyne lidar. II. Correlogram accumulation," IEEE Trans. Geo. Sci. Remote Sensing 31, 28-35 (1993).

14. D. S. Zmic, "Estimation of spectral moments of weather echoes," IEEE Trans. Geosci. Electronics $G E-17,(1979)$.

15. Doviak, R. J., and D. S. Zmic, 1984: second edition 1993: Doppler radar and weather observations, Academic Press 
16. Van Trees, H. L., 1968: Detection, Estimation, and Modulation Theory, Part I, John Wiley and Sons, Inc.

17. Helstrom, C. W., 1968: Statistical theory of signal detection, Pergamon Press.

18. R. G. Frehlich, "Cramer-Rao Bound for Gaussian random processes and applications to radar processing of atmospheric signals," IEEE Trans. Geo. Sci. Remote Sensing (in press), (1993).

19. R. G. Frehlich and M. J. Kavaya, "Coherent laser radar performance for general atmospheric refractive turbulence," Appl. Opt. 30 5325-5352 (1991).

20. Monin, A. S., and A. M. Yaglom, Statistical Fluid Mechanics: Mechanics of Turbulence, Volume 2, MIT Press.(1975)

21. J. H. Chumside and H.T. Yura, "Speckle statistics of atmospherically backscattered laser light," Appl. Opt. 22, 2559-2565 (1983).

22. Barry J. Rye, "Spectral correlation of atmospheric lidar retums with range-dependent backscatter," J. Opt. Soc. Am. A. 7, 2199-2207 (1990). 
Figure 1

Fig.1 Effects of wind shear on normalized signal covariance [Eqs. (6), (9), (38), $F=0$ ] as a function of $\tau / \tau_{P}$ with constant SNR over the observation interval and $\left(\stackrel{\longrightarrow}{\perp} \mu=t_{0}\right.$, no wind shear, (...) $\mu-t_{0}=\sigma$ and $\tau_{P}=\tau_{W S} ;(--) \mu-t_{0}=2 \sigma$ and $\tau_{P}=\tau_{W S} ;(-..) \mu-t_{0}=1 \sigma$ and $\tau_{P}=2 \tau_{W S} ;$ and $(. .-. .-)$ $\mu-t_{0}=2 \sigma$ and $\tau_{P}=2 \tau_{W S}$.

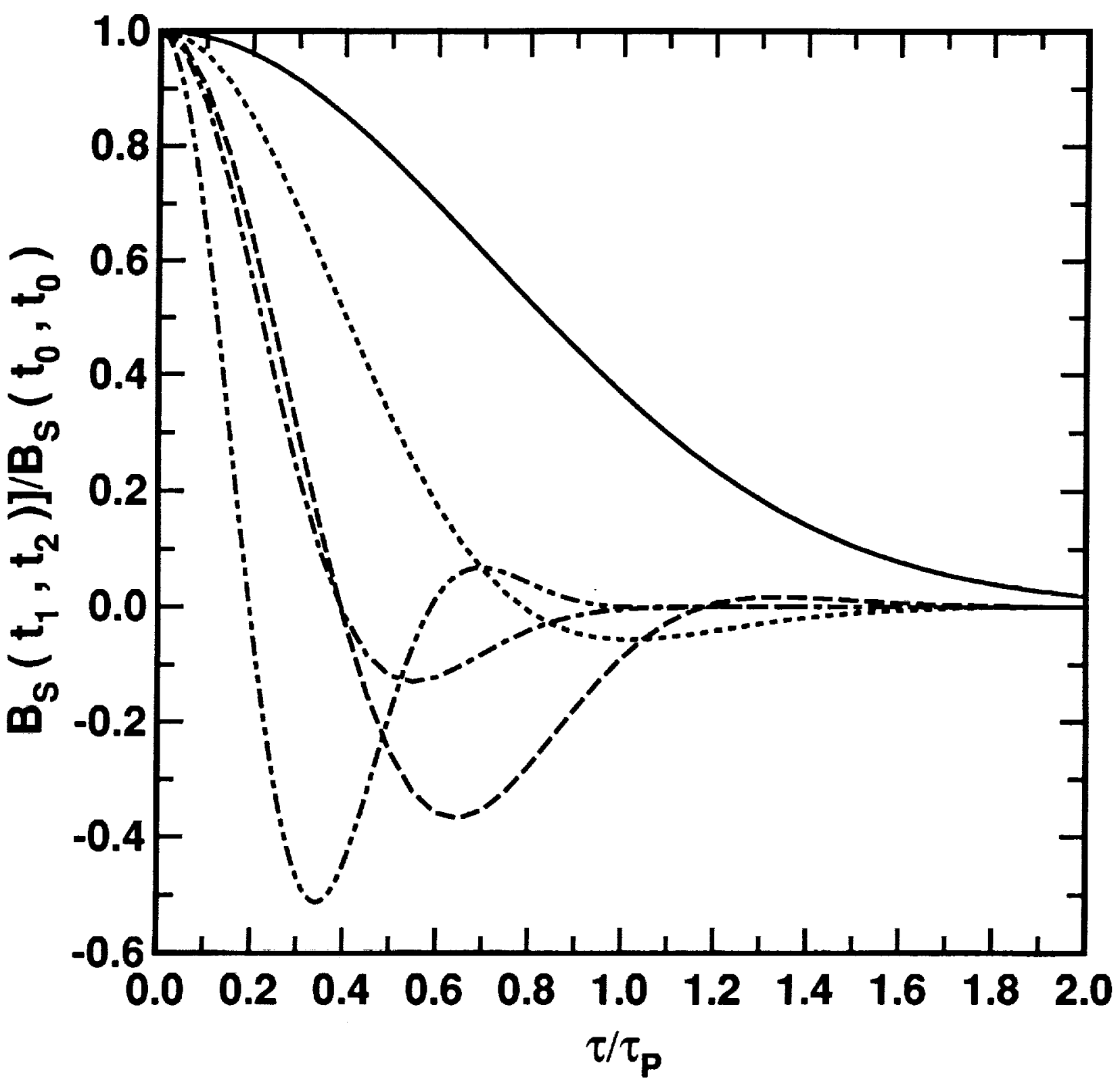


Fig.2 Effects of wind shear and gradients in SNR over the observation interval on the normalized signal covariance [Eqs. (6), (9), (38), $F=0$ ] as a function of $\tau / \tau_{p}$ compared with constant SNR over the observation interval and no wind shear $\left(\longrightarrow\right.$. The curves represent $(\ldots) \mu-t_{0}=\sigma, \tau_{P}=\tau_{W S}$, and $\gamma=0.2 ;(--) \mu-t_{0}=\sigma, \tau_{P}=\tau_{W S}$, and $\gamma=0.4 ;(-..) \mu-t_{0}=2 \sigma, \tau_{P}=2 \tau_{W S}$, and $\gamma=0 ;(. .-.$. $\mu-t_{0}=2 \sigma, \tau_{P}=2 \tau_{W S}$, and $\gamma=0.2$; and $(. .-. .--) \mu-t_{0}=2 \sigma, \tau_{P}=2 \tau_{W S}$, and $\gamma=0.4$.

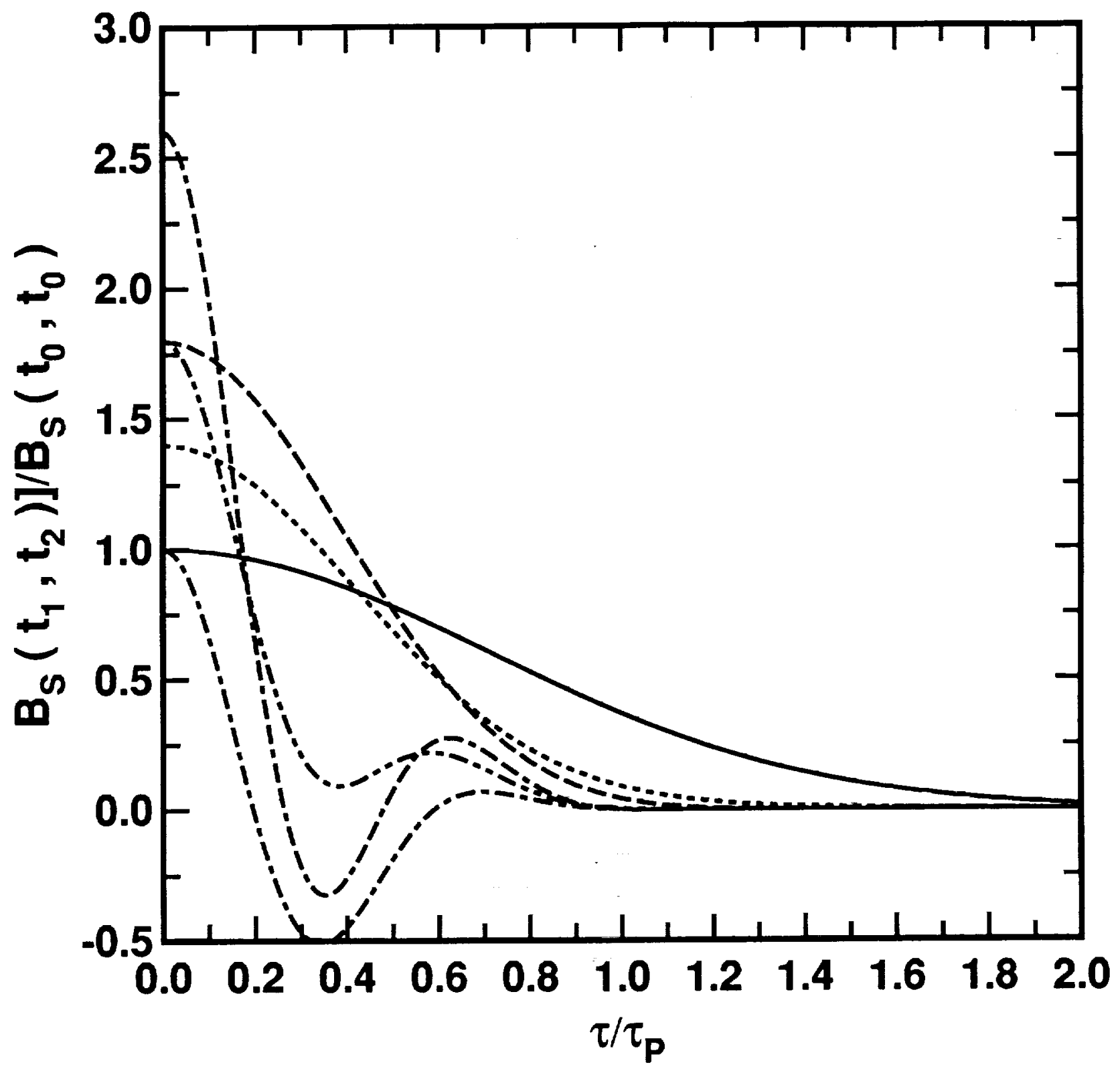


Fig.3 Effects of the finite transverse dimensions of a lidar beam and wind turbulence on the normalized signal covariance [Eqs. (6), (9), (49), $F=0$ ] as a function of $\tau / \tau_{P}$ with constant SNR over the observation interval and case a) $\mu=t_{0}$ with $\left(\Longrightarrow\right.$ no wind turbulence; $(\ldots) \Lambda=2$, and $\sigma_{B} \ll \Delta r$; (- -) $\Lambda=2$, and $\sigma_{B}=\Delta r / 2 ;(. .-) \Lambda=2$, and $\sigma_{B}=\Delta r ;(-. ..) \Lambda=2$, and $\sigma_{B}=2 \Delta r ;$ and case b) the same as case a) but with $\mu-t_{0}=\sigma$.

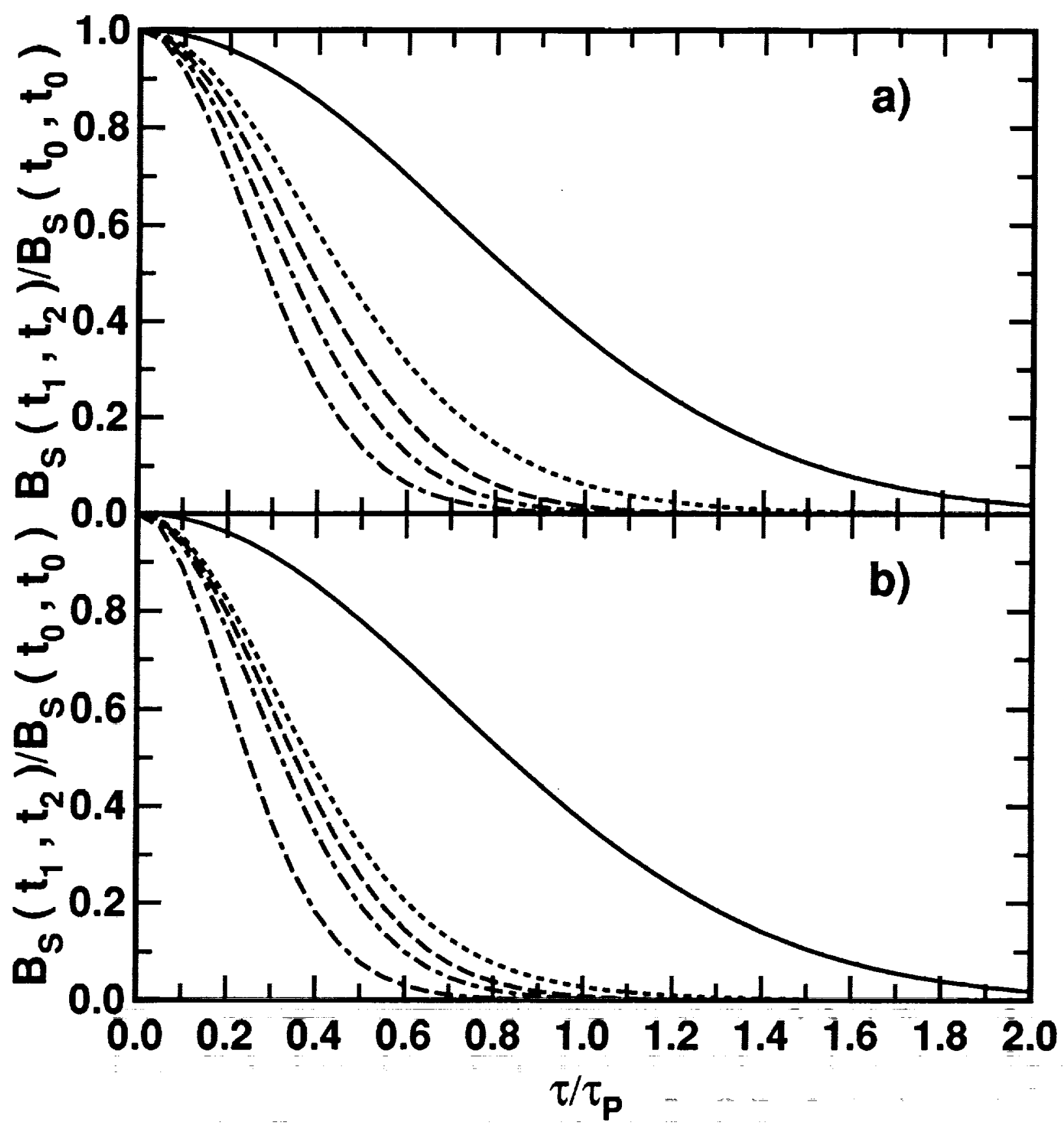


Figure 4

Fig.4 Effects of wind turbulence on the normalized signal covariance [Eqs. (6), (9), (50) $F=0$ ] as a function of $\tau / \tau_{P}$ with constant SNR over the observation interval and narrow transmitted beam compared to the range resolution $\left(\sigma_{B} \ll \Delta r\right.$ ) for case a) $\mu=t_{0}$ with $({ })$ no wind turbulence; (...) $\Lambda=2 ;(--) \Lambda=4$; and case $b$ ) is the same as case a) but with $\mu-t_{0}=\sigma$.

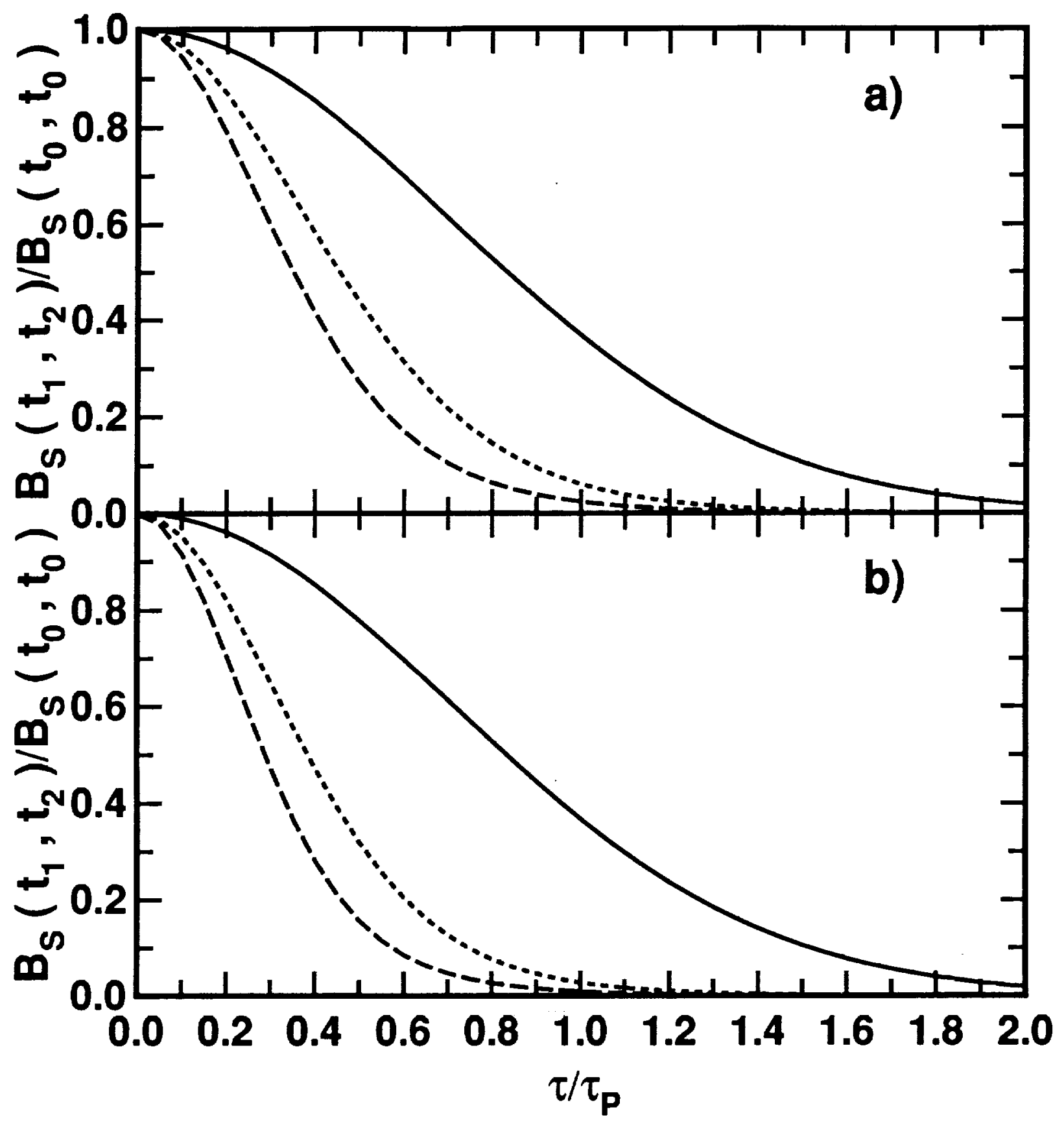


